\title{
Planet destruction and the shaping of planetary nebulae
}

\author{
L. A. Boyle and M. P. Redman \\ Centre for Astronomy, National University of Ireland, Galway, \\ University Road, Galway, Ireland \\ email: 1.boyle2@nuigalway.ie \\ email: matt.redman@nuigalway.ie
}

\begin{abstract}
The shaping of PNe as a result of an interaction with a planet is a hypothesis that has been suggested for nearly two decades. However, exploring the idea observationally is challenging due to the lack of capabilities needed to detect any evidence of such a scenario. Nonetheless, we propose that the hypothesis can be indirectly tested via a combination of exoplanet formation and evolution theories, the star and planet formation histories of the galaxy and the tidal evolution of star-planet systems. We present a calculation of the fraction of planetary nebulae in the galaxy today which have undergone an interaction with a planet, concluding that a significant number of visible planetary nebulae may have been shaped by a planet.
\end{abstract}

Keywords. Planetary Nebulae: general, Exoplanets

\section{Introduction}

One of the fundamental objectives still present in planetary nebulae (PNe) studies is to establish a characterisation of the mechanisms behind the array of morphologies observed. It is likely that central star binarity is the origin of the majority of bipolar planetary nebulae (Miszalski et al. 2009; Corradi et al. 2014; Jones et al. 2015). However, the period distribution of binary progenitor systems suggests that binary shaping is insufficient to account for the total population of non-spherical PNe (Raghavan et al. 2010). An encouraging idea is that a subset of them may be a result of the interaction of planets with single AGB progenitors (Soker 1996; De Marco \& Soker 2011).

Orbital evolution calculations of planets around evolving stars have yielded crucial insights into the effects of mass-loss and stellar tides on a planets survival (Villaver \& Livio 2007; Kunitomo et al. 2011; Nordhaus \& Spiegel 2013; Madappatt et al. 2016). Given the correct combination of stellar mass, planet mass and initial semi-major axis, tidal forces can dominate over mass-loss and draw the planet into the envelope of its host. Whether the planet enters the envelope on the RGB or the AGB is crucial, since only an AGB interaction can result in the shaping of PNe. However, a planet entering the envelope of an RGB star can have significant consequences as it may impede the evolution of its host, ultimately preventing the formation of a PN at all (Nelemans 2010; Bear \& Soker 2011). In the event that a planet enters the AGB envelope, its angular momentum and orbital energy will be deposited into the star leading to a possible inspiral, destruction and shaping event (Nordhaus \& Blackman 2006).

Unfortunately obtaining direct observational evidence for the planet shaping hypothesis is beyond the reach of current capabilities. The closest thing we have to observational evidence of a planet interaction in a $\mathrm{PN}$ is the suggestion that the presence of metal-rich components embedded within the normal nebular gas can provide a resolution to the long-standing abundance discrepancy problem (Wesson et al. 2005; Yuan et al. 2011). 
The origin of such metal-rich components is still subject to debate, but one possibility put forward is that they may be the remnant of a destroyed planet (Liu et al. 2006; Corradi et al. 2015). In the absence of conclusive evidence we are still limited to a theoretical exploration, as obtaining an observational planet fraction to compliment the close binary fraction is challenging, if not yet impossible. Nonetheless, it is possible to make a prediction on the planet fraction based on our knowledge of the currently known exoplanets. We present a method to estimate the fraction of PNe which could have been shaped by the interaction of an AGB star with a planet.

\section{Estimating the planet fraction of PNe: The known exoplanets}

A primitive estimation for the planet-fraction can be sought by first looking to the distribution of currently known exoplanets. We downloaded information for the known exoplanets from the Exoplanet Orbit Database (exoplanets.org), and only included planetary systems which had been discovered, or followed up with, radial velocity measurements (thus where the star and planet masses and semi-major axes were available). This consisted in a total of 670 stars. The semi-major axis evolution of each planet system is modelled using the equations of orbital evolution around evolving stars given by

$$
\dot{\mathrm{a}}=\mathrm{a} \frac{\dot{\mathrm{M}}_{*}}{\mathrm{M}_{*}}-\mathrm{f} \frac{\mathrm{a}}{\tau_{\text {conv }}} \frac{\mathrm{M}_{\mathrm{env}}}{\mathrm{M}_{*}}\left(1+\frac{\mathrm{M}_{\mathrm{pl}}}{\mathrm{M}_{*}}\right) \frac{\mathrm{M}_{\mathrm{pl}}}{\mathrm{M}_{*}}\left(\frac{\mathrm{R}_{*}}{\mathrm{a}}\right)^{8}
$$

The first term in (2.1) represents the evolution due to mass-loss of the star, where $\mathrm{M}_{\mathrm{pl}}$, $\mathrm{M}_{*}, \dot{\mathrm{M}}_{*}$ are the planet and stellar masses and the stellar mass-loss rate, respectively, and $\mathrm{a}$ is the semi-major axis of the planet. The second term in (2.1) is the evolution due to tides (Zahn 1977), where $\mathrm{M}_{\text {env }}$ is the mass of the stellar envelope, $\mathrm{R}_{*}$ is the stellar radius and $\tau_{\text {conv }}$ is the convective timescale. The stellar parameters are calculated using the MESA stellar evolution code (Paxton et al. 2015). With the assumptions that all stars have a planet and that the stellar population is a good proxy for the PN progenitor population, we can make a simple estimation for the fraction of PN progenitors having interacted with a planet. To do this we categorise the hypothetical population as follows:

- Systems which can shape a PN

(a) Single stars interacting with a massive planet on the AGB

(b) Binary systems with a common envelope on the AGB

(c) Binary systems with no common envelope, but having a mild interaction

- Non-interacting systems

(a) Single stars with no interaction

(b) Binary systems with large separations

- Systems which may prevent a PN

(a) Single stars interacting with a massive planet on the RGB

(b) Binary systems with a common-envelope on the RGB

We do not model the orbital evolution of binary systems, instead we adopt the period distribution of Raghavan et al. (2010) who estimate that $\approx 50 \%$ of stars form in binaries, where $\approx 30 \%$ can undergo an interaction ( $14 \%$ on RGB, $16 \%$ on AGB).

The resulting population estimates are presented in Table 1. While it may appear that the majority of planet interactions occur on the RGB, we still do not know the true massperiod distribution of exoplanets. The radial velocity method is inherently biased towards the most close-in massive planets. This results in an apparent lack of planets with larger intitial separation capable of avoiding an interaction on the RGB, thus being available for an interaction on the AGB. Moreover, this analysis is based on the premise that the 
Table 1. Population estimates based on exoplanet data.

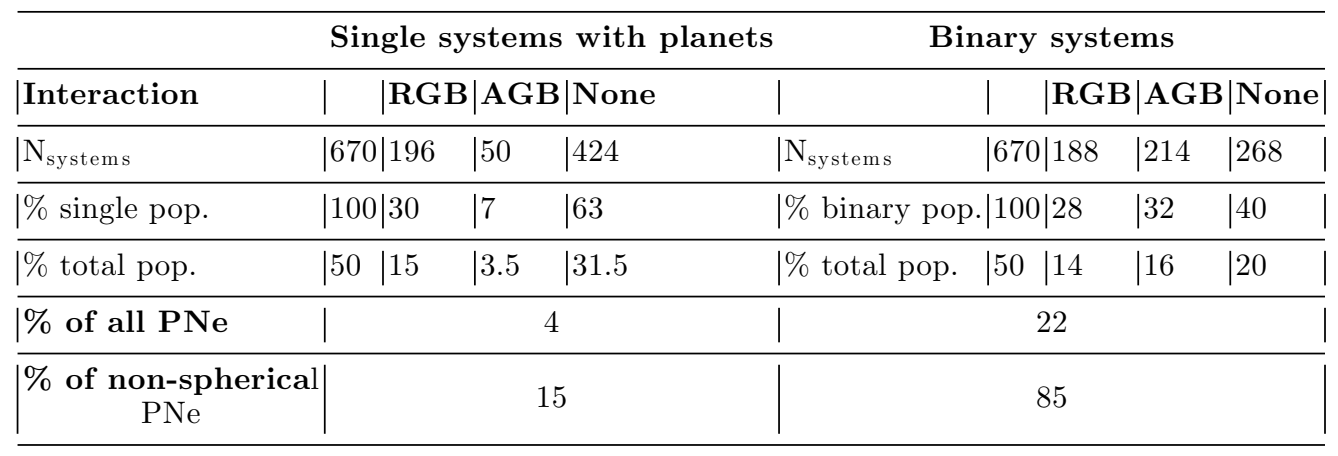

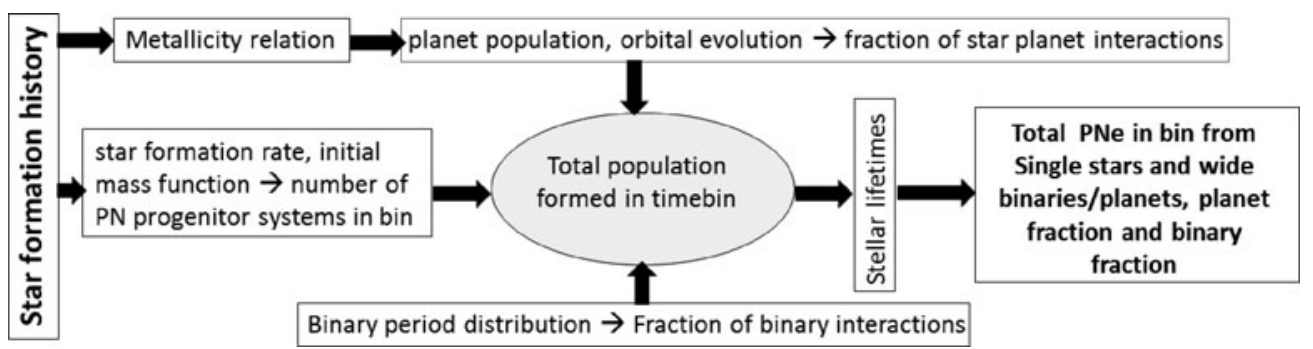

Figure 1. Simplified work-flow of a single time-bin in our model galaxy.

total population of PNe in the galaxy today evolved from a similar stellar population to that of the observed exoplanet hosts. For this assumption to hold true the galaxy would need to be a coeval population. In Section 3 we present a more robust approach.

\section{Estimating the planet fraction of PNe: A galactic inventory}

Since our aim is to predict the fraction of PNe in the galaxy whose progenitors would have suffered an interaction with a planet, we need to model the star-planet formation and evolution history. Our model galaxy is split into time-bins pre-determined by the star formation history provided by Behroozi et al. (2013). In each time-bin we calculate the total number of PNe progenitors which would have evolved to, and are currently still visible in, the present day galaxy by employing the star formation rate, the stellar initial mass function and stellar lifetimes. Combining the total number of PNe progenitor systems in each bin with a synthetic planet mass-period distribution (Ida \& Lin 2005; Mordasini et al. 2015), our orbital evolution model described previously and the fraction of stars that have a planet (Gaidos \& Mann 2014), we can determine the number of PNe from that time-bin that had a planet interaction. The criteria for systems resulting in non-spherical PNe for planets and binaries are the same as those outlined in Section 2. Finally, we sum the contributions from each bin allowing us to arrive at the fractions of PNe which have evolved via interactions with a planet, a binary or with no interaction. See Figure 1, and Boyle \& Redman (2017, in prep).

\section{Results and Implications}

Our model predicts a total PN population of $\approx 6 \times 10^{4}$, of which $\approx 3.5 \times 10^{3}(6 \%)$ would have evolved via an interaction with a massive planet, and $\approx 12 \times 10^{3}(20 \%)$ via a 
binary interaction. This result suggests a total fraction of non-spherical PNe of $\approx 26 \%$, translating to $\approx 23 \%$ and $\approx 77 \%$ of all non-spherical PNe having a planetary and binary origin, respectively. Moe \& De Marco (2012) performed a thorough population synthesis of PNe from stellar binaries reporting a binary fraction of $\approx 70 \%$, which provides us with a benchmark on which to compare our results. Our model predicts a total number of PNe discrepant with their result of $8,100 \pm 2,300$, however this is likely due to differing prescriptions for the star formation history, along with simplifications of our model. We anticipate that when appropriate refinements are made, and uncertainties taken into account that our resulting PN population and binary statistic will be in agreement.

While our model can still be regarded as preliminary, our result suggests that a significant fraction of PNe progenitors undergo interactions with orbiting massive planets. These interactions can potentially affect the morphology of the resulting PNe, complimenting the binary fraction. Additionally, this result provides support for the hypothesis that planetary debris leftover from a destruction event could be the source of metal-rich gas leading to abundance discrepancies in some PNe. We conclude that the planet-shaping hypothesis is a plausible scenario and warrants further investigation.

L. A. Boyle acknowledges support by the Irish Research Council under their postgraduate research scheme, and the IAU for travel funding. We thank the following for helpful discussions: N. Madappattu, O. De Marco, E. Villaver, J. Nordhaus, N. Soker.

\section{References}

Bear, E. \& Soker, N. 2011, MNRAS, 411, 1792

Behroozi, P. S., Wechsler, R. H., \& Conroy, C. 2013, ApJ, 770, 57

Corradi, R. L. M., Rodríguez-Gil, P., Jones, D., et al. 2014, MNRAS, 441, 2799

Corradi, R. L. M., García-Rojas, J., Jones, D., \& Rodríguez-Gil, P. 2015, ApJ, 803, 99

De Marco, O. \& Soker, N. 2011, PASP, 123, 402

Gaidos, E. \& Mann, A. W. 2014, ApJ, 791, 54

Ida, S. \& Lin, D. N. C. 2005, ApJ, 626, 1045

Jones, D., Boffin, H. M. J., Miszalski, B., et al. 2014, A\&AP, 562, A89

Jones, D., Boffin, H. M. J., Rodríguez-Gil, P., et al. 2015, A\&AP, 580, A19

Kunitomo, M., Ikoma, M., Sato, B., Katsuta, Y., \& Ida, S. 2011, ApJ, 737, 66

Liu, X.-W., Barlow, M. J., Zhang, Y., Bastin, R. J., \& Storey, P. J. 2006, MNRAS, 368, 1959

Madappatt, N., De Marco, O., \& Villaver, E. 2016, MNRAS, 463, 1040

Miszalski, B., Acker, A., Moffat, A. F. J., Parker, Q. A., \& Udalski, A. 2009, A $ॄ A P, 496,813$

Moe, M. \& De Marco, O. 2012, IAU Symposium, 283, 111

Mordasini, C., Mollière, P., Dittkrist, K.-M., Jin, S., \& Alibert, Y. 2015, IJA, 14, 201

Nelemans, G. 2010, ApESSS, 329, 25

Nordhaus, J., \& Blackman, E. G.2006, MNRAS, 370, 2004

Nordhaus, J. \& Spiegel, D. S. 2013, MNRAS, 432, 500

Paxton, B., Marchant, P., Schwab, J., et al. 2015, ApJS, 220, 15

Raghavan, D., McAlister, H. A., Henry, T. J., et al. 2010, ApJS, 190, 1

Soker, N. 1996, ApJL, 460, L53

Villaver, E. \& Livio, M. 2007, ApJ, 661, 1192

Wesson, R., Liu, X.-W., \& Barlow, M. J. 2005, MNRAS, 362, 424

Yuan, H.-B., Liu, X.-W., Péquignot, D., et al. 2011, MNRAS, 411, 1035

Zahn, J.-P. 1977, A\&SAP, 57, 383 